\title{
Philosophiques
}

\section{Pourquoi le grand nombre est plus intelligent que le petit nombre, et pourquoi il faut en tenir compte}

\section{Hélène Landemore}

Volume 40, numéro 2, automne 2013

Autorité démocratique et contestation

URI : https://id.erudit.org/iderudit/1023698ar

DOI : https://doi.org/10.7202/1023698ar

Aller au sommaire du numéro

\section{Éditeur(s)}

Société de philosophie du Québec

ISSN

0316-2923 (imprimé)

1492-1391 (numérique)

Découvrir la revue

Citer cet article

Landemore, H. (2013). Pourquoi le grand nombre est plus intelligent que le petit nombre, et pourquoi il faut en tenir compte. Philosophiques, 40(2),

283-299. https://doi.org/10.7202/1023698ar
Résumé de l'article

Cet article présente les bases d'un argument épistémique en faveur de la démocratie définie comme procédure de décision collective. Il explore également les implications d'un tel argument épistémique par rapport à d'autres justifications établies de la démocratie, par rapport aux explications scientifiques de ses succès empiriques, et en termes de politiques publiques à mener. En ce qui concerne l'argument épistémique proprement dit, il repose sur le concept de "raison démocratique ", autrement dit l'intelligence collective des individus dans le domaine politique, et propose, de manière contre-intuitive, que la raison démocratique est davantage tributaire de la diversitécognitive des individus qui prennent par aux décisions que de leurs aptitudes personnelles. Généralement, l'argument de la raison démocratique complète les arguments procéduraux basés sur l'équité et l'égalité pour offrir une explication fonctionnaliste complète de la démocratie. Pour finir, cet article défend l'idée de réformes institutionnelles favorisant la participation citoyenne dans le processus de prise de décision collective. 


\title{
Pourquoi le grand nombre est plus intelligent que le petit nombre, et pourquoi il faut en tenir compte
}

\author{
HÉLÈNE LANDEMORE \\ Université Yale \\ helene.landemore@yale.edu
}

\begin{abstract}
RÉSUMÉ. - Cet article présente les bases d'un argument épistémique en faveur de la démocratie définie comme procédure de décision collective. II explore également les implications d'un tel argument épistémique par rapport à d'autres justifications établies de la démocratie, par rapport aux explications scientifiques de ses succès empiriques, et en termes de politiques publiques à mener. En ce qui concerne l'argument épistémique proprement dit, il repose sur le concept de « raison démocratique», autrement dit l'intelligence collective des individus dans le domaine politique, et propose, de manière contre-intuitive, que la raison démocratique est davantage tributaire de la diversitécognitive des individus qui prennent par aux décisions que de leurs aptitudes personnelles. Généralement, l'argument de la raison démocratique complète les arguments procéduraux basés sur l'équité et l'égalité pour offrir une explication fonctionnaliste complète de la démocratie. Pour finir, cet article défend l'idée de réformes institutionnelles favorisant la participation citoyenne dans le processus de prise de décision collective.
\end{abstract}

\begin{abstract}
This paper presents the foundations of a systematic epistemic case for democracy as a collective decision-rule and explores the implications of this epistemic claim for normative justifications of democracy, scientific explanations of its empirical success, and policy reforms. As far as the epistemic case is concerned, the paper proposes an account based on the concept of "democratic reason," or the collective intelligence of the people in politics. The paper argues that, counter-intuitively, democratic reason is more a function of the cognitive diversity of the individuals taking part in the decision than of their individual ability. As an account of democracy's epistemic benefits, the argument from democratic reason supplements procedural accounts based on fairness and equality to provide a complete functionalist explanation of democracy. Finally, the argument supports policy reforms increasing citizens' participation in the collective decision-process.
\end{abstract}

L'idée de sagesse collective - c'est-à-dire l'idée selon laquelle on est plus intelligent à plusieurs, et qu'en général plusieurs têtes valent mieux qu'une - est au moins aussi ancienne que les Politiques d'Aristote ${ }^{1}$. On pourrait dire que cette idée se trouve au cœur de toute entreprise collective, et peut-être même, de toute société. Les individus se rassemblent, parce qu'ils savent qu'ils peuvent obtenir davantage ensemble qu'ils ne le pourraient individuellement, et ils prennent des décisions de manière collective

1. Voir Waldron, I995, pour un commentaire plus complet de ce qu'il a appelé, de manière particulièrement heureuse, la «doctrine de la sagesse de la multitude » ou DSM.

PHILOSOPHIQUES 40/2 - Automne 2013, p. 283-299 
parce qu'ils croient que, du point de vue qualitatif, de telles décisions se révèleront en moyenne plus intelligentes que ne se révèleraient celles que chacun d'entre eux prendrait par lui-même. La position inverse, qui dénonce la «folie des foules» (Charles Mackay, I84I) et affirme que «trop de cuisinières gâtent la sauce ", est cependant tout aussi commune. Dans les sciences politiques, depuis Platon jusqu'aux démocrates élitistes contemporains, l'idée que la multitude est incapable de se comporter de manière autonome, sans même parler de son inaptitude à prendre quelque décision avisée que ce soit, est sans doute celle qui domine ${ }^{2}$. Voilà qui est assez paradoxal, étant donné cette autre croyance communément acceptée, et selon laquelle la démocratie est la seule forme de gouvernement légitime (et pas juste le pire régime à l'exception de tous les autres).

Ces dernières années, cependant, l'idée d'intelligence collective appliquée aux sociétés a connu un regain d'intérêt à la faveur de travaux comme ceux de Howard Rheingold avec Foules intelligentes (2005), James Surowiecki avec La sagesse des foules (2008), ou Cass Sunstein avec Infotopia: How Many Minds Produce Knowledge. En parallèle, mais de manière indépendante, la théorie démocratique a vu un petit groupe d'individus auto-baptisés « démocrates épistémiques » reprendre à son compte, en général au sein du paradigme dominant de la "démocratie délibérative", l'idée d'autorité démocratique et celle de la capacité des institutions démocratiques à produire de bonnes décisions collectives (par exemple Estlund, I 997 et 2008 1; Goddin, 2003 et 2008). Dans le même temps, les théoriciens formalistes ont redécouvert le théorème du jury de Condorcet. La littérature qui a dérivé de ces deux sources s'est rapidement intéressée à la question de sa pertinence pour la démocratie (voir par exemple les débats qui ont eu lieu dans Grofman, Feld et Waldron, I989). Plus récemment encore, Josiah Ober a soutenu que l'Athènes de l'Antiquité disposait de propriétés épistémiques qui l'ont rendue capable de collecter et de traiter l'information de manière plus efficace que toutes les cités-États rivales, telles que Sparte (Ober, 2010).

Il y ainsi eu un retour en grâce de la vieille idée aristotélicienne selon laquelle plusieurs têtes valent mieux qu'une, réhabilitée de plusieurs points de vue. En rapprochant les différents travaux consacrés à cette question et en y ajoutant certains concepts empruntés à la psychologie et aux sciences cognitives, j’ai moi-même proposé une défense épistémique poussée de la démocratie fondée sur l'idée d'intelligence collective (Landemore, 2008, 2002a et 20I3). Dans ce travail, je soutiens la thèse selon laquelle les institutions démocratiques, telles que la délibération inclusive et la règle de la majorité couplée au principe du suffrage universel, allient leurs propriétés épistémiques de manière à transformer le plomb de la contribution individuelle des

2. Pour une version récente de ce vieux thème platonicien, voir Caplan, 2007. 
citoyens en l'or de la "raison démocratique $»^{3}$ et fournissent à la démocratie un avantage épistémique sur toute variante de l'oligarchie. Cette affirmation va plus loin que la conjecture timide avancée par David Estlund, selon laquelle la démocratie vaudrait épistémiquement mieux que le hasard, autrement dit, qu'elle serait plus susceptible de parvenir à la vérité qu'une procédure reposant sur le hasard, comme le fait de tirer à pile ou face quand on doit faire un choix entre deux options (Estlund, 2008). Elle prolonge également un argument proposé par John Stuart Mill en faveur de la supériorité du gouvernement représentatif sur toute forme d'aristocratie et de monarchie (Mill, 20 го [I 86I], chap. V) ${ }^{4}$. On peut enfin l'interpréter comme une version plus générale de la thèse de l'agrégation de la connaissance avancée par Ober.

Cet article se propose d'accomplir deux choses. La première est de présenter et défendre, sous forme condensée, l'idée qu'il existe un lien théorique entre le phénomène de l'«intelligence collective» ou de la «sagesse collective» d'une part, et le principe démocratique de la prise de décision collective d'autre part. La raison pour laquelle on peut s'attendre à ce que les plus nombreux soient plus intelligents que les moins nombreux réside, selon moi, dans le fait qu'il y a probablement une corrélation entre la prise de décision inclusive et la présence d'un élément dont il a récemment été démontré qu'il joue un rôle clé dans l'émergence de l'intelligence collective, à savoir la "diversité cognitive» (Hong and Page, 200I, 2004, 2009; Page, 2007). Dans la mesure où ce type de diversité s'accroît de manière caractéristique lorsque l'on inclut davantage d'individus, et toutes choses étant égales par ailleurs (c'est-à-dire, si l'on fait abstraction d'un certain nombre de facteurs d'interférences, comme les coûts de communication), les plus nombreux doivent être plus intelligents (Landemore, 20I2a, 20 I 2b, 20I3).

Dans un second temps, cet article se penche sur les implications positives et normatives de l'idée d'intelligence collective dans la perspective d'une défense de la démocratie. Sur le plan des implications positives, je soutiens la thèse selon laquelle l'idée de "raison démocratique » — ou d'intelligence collective du peuple en matière politique - fournit un cadre conceptuel adéquat pour un certain nombre de recherches empiriques en sciences politiques et en économie, du moins le type de recherches qui visent à identifier des corrélations entre certains résultats considérés comme désirables, et la prise de décision démocratique. Il me semble également que l'idée d'une

3. C'est ainsi que je propose d'appeler l'intelligence collective que produisent les institutions démocratiques.

4. Mill pensait que le véritable risque pour la démocratie, ou, comme il l'appelle, le gouvernement représentatif, n'était pas de se trouver comparé à la règle d'un seul ou de quelquesuns, mais d'être comparé à une bureaucratie. Si l'on veut être tout à fait juste, il faut reconnaître que la manière dont il comprenait le fonctionnement du gouvernement représentatif (et en particulier des assemblées représentatives) diverge dans une certaine mesure de nos conceptions modernes. Par exemple, il ne pensait pas que la fonction des assemblées représentatives était de faire les lois, mais seulement de sélectionner les experts à qui serait confiée cette tâche. 
intelligence collective du peuple fournit un cadre dans lequel un bon nombre des résultats produits par la recherche sur l'opinion publique pourraient trouver une interprétation moins décourageante qu'elle ne l'est d'ordinaire. Sur le plan de la théorie démocratique normative, il me semble que l'argument à tirer de l'intelligence collective peut non seulement s'ajouter au répertoire déjà existant des arguments en faveur de la démocratie, mais nous invite également à nous intéresser au statut de ces autres arguments. La conclusion, enfin, présente quelques réflexions sur les implications potentielles de l'argument de l'intelligence collective en ce qui concerne les politiques à mener. Jesuggère que, là où ils sont possibles et conduits de la bonne manière, les efforts en vue de rendre le processus de prise de décision collective plus inclusif et plus ouvert à la participation citoyenne devraient aussi permettre de parvenir à des décisions collectives non seulement plus justes, mais aussi, dans l'ensemble, plus intelligentes.

\section{La raison démocratique: l'argument de l'intelligence collective}

Je commencerai par donner une première définition de l'idée de sagesse collective, ou d'intelligence collective, que j'applique au domaine de la politique démocratique sous le nom de "raison démocratique». Je conçois la raison démocratique comme un certain type de phénomène d'émergence, celui en vertu duquel un peuple se révèle être plus intelligent ou plus sage que les individus qui le composent. Dans cet article, j'utiliserai indifféremment les termes «intelligence » et «sagesse», bien que le concept de sagesse soit plus riche que celui d'intelligence, puisqu'il recouvre les notions d'expérience, de connaissance éprouvée par le temps et, de façon plus générale, celle d'intelligence diachronique - autant d'aspects qui relèvent sans doute de la raison démocratique, mais sur lesquels je n'insisterai pas ici ${ }^{5}$. Le seul aspect diachronique de la raison démocratique dont nous dirons un mot dans cet article est celui introduit par l'institution de la représentation politique, dans la mesure où elle crée une forme de médiation temporelle entre l'apport des citoyens et sa traduction dans des politiques effectives.

L'argument substantiel que je propose en faveur de la démocratie, en liaison avec cette idée de raison démocratique, peut être résumé dans la simple affirmation suivante: la démocratie constitue une bonne procédure de prise de décision collective parce que, entre autres et toutes choses étant égales par ailleurs, elle maximise nos chances collectives de faire les bons choix $^{6}$. De plus, la démocratie dispose d'une supériorité épistémique sur les autres procédures de prise de décision qui sont moins inclusives en raison du

5. Pour une étude plus complète de ces notions, voir par exemple l'article de D. Andler dans Landemore and Elster, $20 \mathrm{I} 2$.

6. Laissant pour l'instant de côté la question philosophique épineuse de savoir ce qu'est un «bon» choix, on accepte l'idée qu'il existe un tel standard de correction indépendant de toute procédure (ce que requiert tout approche épistémique de la démocratie). 
lien essentiel qui existe entre le caractère plus inclusif d'un processus de prise de décision, d'une part, et d'autre part, quelque chose qui s'est révélé être un élément clé de l'intelligence collective, à savoir la "diversité cognitive".

Sans simplifier outrancièrement les choses, on peut dire que le phénomène de l'intelligence collective est le produit de deux facteurs. Le premier renvoie à l'aptitude ou à l'intelligence des membres du groupe pris individuellement, et que l'on peut exprimer sous la forme d'une aptitude moyenne. Appelons-la leur "QI». Ce facteur renvoie à la propriété d'une "moyenne » idéale, ou d'un membre du groupe qui pourrait être considéré comme «représentatif». L'autre facteur est celui de la «diversité cognitive» ou, en gros, l'existence de différentes manières de voir le monde, de l'interpréter, et d'y appliquer des modèles prédictifs, au sein d'un même groupe. Plus techniquement, la diversité cognitive renvoie à une diversité de perspectives (la manière de se représenter les situations et les problèmes), à une diversité d'interprétations (la manière d'élaborer des solutions en réponse à ces problèmes), et à une diversité de modèles prédictifs (la manière d'inférer les causes et les effets) (Page, 2007: 7; voir également Stich, I999). Remarquons que la diversité cognitive est une propriété du groupe lui-même, et non d'un individu donné en son sein.

Hong et Page montrent que l'aptitude individuelle et la diversité cognitive jouent un rôle différent selon les contextes - une intuition que nous pouvons tous saisir, mais qu'ils élèvent au rang de vérité mathématique. Dans les situations de résolution de problèmes, ce qui compte le plus pour la qualité de la réponse collective n'est pas tant l'intelligence des individus pris un par un, mais la diversité cognitive au sein de leur groupe (théorème de l'avantage de la diversité sur l'aptitude). Pour une illustration rapide et une mise en situation de cette idée (qui se trouve développée plus avant dans Landemore, 20I $2 \mathrm{a}$ et $20 \mathrm{I} 3$ ), on peut songer au processus de décision tel qu'il se déroule dans Douze hommes en colère. Individuellement, tous les jurés initialement convaincus de la culpabilité du prévenu se seraient enferrés dans un jugement erroné. Même le juré qui s'écarte initialement du consensus (incarné par Henri Fonda) n'aurait pas été, seul, en mesure de fonder son intuition vague qu'on pouvait raisonnablement douter de la culpabilité du prévenu. Ce n'est qu'après avoir soumis l'ensemble des informations et des arguments disponibles à la discussion générale, les avoir passés au crible et examinés sous les différentes loupes du groupe que les membres du jury parviennent de manière unanime et assurée à un verdict de non-culpabilité.

L'idée générale ainsi illustrée dans ce film est que ce qui compte le plus pour la résolution de problèmes, par exemple, ceux auxquels ce jury devait faire face, est, plus que le degré d'intelligence respectif de chacun des membres du groupe, la variété des manières de penser qui existent en son 
$\operatorname{sein}^{7}$. Après tout, au départ, dix des onze jurés ont tort — ils présentent un seuil de QI collectif très bas, si l'on veut. Pourtant, et ce en vertu d'une différence initiale de perspective sur la question (exprimée par le juré numéro 8), qui donne lieu à une discussion sur les raisons que chaque juré pourrait avoir de choisir le verdict de culpabilité, des perspectives différentes sont adoptées, lesquelles apportent de nouveaux éléments. En définitive, les jurés se montrent capables de se guider les uns les autres vers la bonne réponse. Autrement dit: un minimum de diversité cognitive peut compenser bien des incompétences individuelles.

Dans d'autres contextes, cependant, la diversité cognitive a autant d'importance que les aptitudes individuelles (théorème de la diversité). Ces contextes renvoient aux situations dans lesquelles on agrège simplement les jugements individuels en une réponse collective, sans qu'il soit possible de séparer les bonnes contributions des mauvaises, à la différence de ce qui se produit dans un processus diachronique et délibératif de résolution de problème comme celui qu'illustre l'exemple du jury (théorème de l'avantage de la diversité sur l'aptitude). Autrement dit, dans les contextes de pure agrégation (plutôt que de délibération), il y a une équivalence stricte entre les deux éléments de l'intelligence collective. Sans expliquer en détail pourquoi l'agrégation des jugements dans un groupe peut permettre de parvenir à des jugements et des prédictions collectifs plus pertinents que toute prédiction ou tout jugement individuels, disons juste que, d'après l'analyse proposée par Hong et Page, cette propriété tient à l'existence de corrélations négatives entre les prédictions de chacun des votants ${ }^{8}$. L'avantage des corrélations négatives de ce type est de garantir que, là où une personne se trompe, une autre personne a plus de chances de faire le bon choix, et inversement. Par conséquent, au total, les erreurs s'annulent de manière systématiquement avantageuse. Dès lors, l'erreur «moyenne » du groupe (c'est-à-dire l'erreur collective à laquelle on parvient, dans les cas où on peut la calculer, par exemple quand il s'agit de prédire des quantités) sera moindre que l'erreur moyenne d'un individu pris au hasard, et en fait elle est d'autant moindre que la différence entre les modèles prédictifs utilisés par les individus est grande (c'est-à-dire, qu'il y a plus de diversité cognitive au sein du groupe).

Pour donner une illustration de ce phénomène, prenons la manière dont les gens font des prédictions pour choisir, entre deux candidats, le meilleur président potentiel. Certains d'entre nous fonderont leur jugement sur sa compétence présumée en matière d'affaires sociales. D'autres feront une prédiction sur la base de son attachement au principe du conservatisme fiscal et sur l'état de l'économie tel qu'il est prévu pour les années à venir. Il y aura probablement des corrélations négatives entre les prédictions de quelqu'un

7. Pour un autre exemple tiré de la politique locale, voir Landemore, 20I 2b.

8. Je laisse au lecteur le soin de se reporter àla démonstration mathématique des théorèmes plus généraux dans Page, 2007 (chap. 8). 
qui se concentre uniquement sur les compétences du candidat en matière sociale, et quelqu'un qui se concentre seulement sur ses compétences en matière de fiscalité. Dès lors, le jugement collectif produit par l'agrégation de modèles prédictifs aussi variés aura tendance à atteindre des prédictions plus exactes que ne le pourrait par lui-même un modèle prédictif individuel. En d'autres termes, il est plus probable que nous élisions le bon président si nous agrégeons les modèles prédictifs des démocrates et des républicains plutôt que de faire des prédictions sur la base des jugements d'un seul de ces groupes.

On pourrait cependant se demander d'où proviennent ces corrélations négatives. Elles proviennent du fait que, quand ils considèrent différents candidats, différents individus se focalisent sur différentes dimensions d'une même qualité (ce que Page appelle «perspective»), ici la compétence relative à la fonction de président. Une personne se concentre sur la compétence sur les questions sociales, une autre sur la compétence en matière économique. Cela produit ce que Page appelle des «interprétations de projection sans chevauchement», c'est-à-dire des interprétations de la compétence du candidat qui ne contiennent aucune variable ou dimension en commun (en l'occurrence, la compétence sur les questions sociales ou économiques) ${ }^{9}$.

Remarquons que, dans leur analyse, Hong et Page mettent l'accent non pas tant sur le grand nombre d'individus que sur la diversité cognitive qu'un tel nombre peut présenter. La beauté de leur explication vient du fait que l'agrégation des jugements individuels peut produire des résultats remarquables, quand bien même il n'y aurait qu'un petit nombre de personnes impliquées. ${ }^{10}$. Selon les auteurs, il n'est pas besoin d'avoir une infinité de votants pour que la règle de la majorité garantisse une exactitude de Ioo p. cent. C'est là un avantage majeur sur les explications de l'intelligence collective qui reposent sur la «loi des grands nombres", comme le théorème du

9. Page formule «la propriété de projection» comme suit: «Si deux individus fondent leurs modèles prédictifs sur différentes variables à partir de la même perspective (formellement, s'ils utilisent une projection qui ne recouvre pas les interprétations), alors la correction de leurs prédictions est corrélée négativement pour les projections binaires» (Page, 2007: 203).

10. Il faut ici ajouter une mise en garde, sans quoi le résultat paraît trop optimiste. On ne peut pas avoir une infinité de variables ou de dimensions associées à une perspective donnée (disons, l'aptitude pour un poste). Quand le nombre de votants devient très élevé, le nombre de variables que les individus utilisent pour faire une prédiction peut rester proportionnellement assez bas (sur les questions sociales et économiques essentielles, les votants peuvent être sensibles au charisme personnel et aux variables de la politique étrangère, mais ils ne prendront plus en considération des variables comme le type de chien que possède la personne, ou son sens de l'humour). Pour éviter les corrélations positives quand le nombre d'individus qui composent la foule s'accroît, les votants doivent soit utiliser des interprétations groupées (cluster interpretations), soit baser leurs interprétations sur des perspectives différentes. L'interprétation d'un votant qui combine des considérations de compétence sur les questions sociales et des considérations de compétence sur les questions financières est un exemple d'interprétations groupées (cluster interpretations). 
jury de Condorcet (TJC - Condorcet, I785) ou l'idée de «miracle de l'agrégation» (Converse, I97I). En effet, puisque que la diversité cognitive peut émerger dès lors qu'il y a plus d'un seul prédicteur, la magie peut alors opérer même dans un petit groupe de trois personnes seulement, et son efficacité s'accroître substantiellement à chaque fois que l'on ajoute une personne au groupe, pour autant que cette personne apporte un modèle prédictif assez distinct (alors que dans le modèle du TJC l'avantage de la règle de la majorité ne se fait sentir qu'à la limite, quand on dispose d'une infinité de personnes, et ajouter un individu au groupe ne fait guère de différence).

Cette analyse, il faut le reconnaître, n'est pas plus à l'abri du risque de biais systématiques que ne le sont le TJC ou la thèse du miracle de l'agrégation. Si les citoyens partagent un certain nombre de conceptions erronées des préjugés racistes, ou les biais systématiques mis en évidence par Bryan Caplan dans les affaires économiques (Caplan, 2007) —, la règle de la majorité ne fera qu'amplifier ces erreurs et, en tout état de cause, rendre les décisions démocratiques moins intelligentes que celle qu'aurait pu réussir à prendre un seul citoyen pris au hasard. Selon Hong et Page, cependant, le risque que la foule soit systématiquement dans l'erreur n'est réel que si l'exactitude prédictive individuelle fait défaut dans le groupe (les gens sont vraiment trop stupides) et que sa manière de faire des prédictions manque de diversité. À celui qui ferait l'hypothèse de votants qui disposent d'un minimum de compétence sur la question en jeu ${ }^{11}$, et d'une société libérale dans laquelle le désaccord et la diversité de pensée sont encouragés, on pourrait répondre que le scénario de Caplan, qui renvoie à la pire des situations où l'erreur moyenne est élevée et la diversité basse - ce qui correspondrait aux conditions du pire des scénarios, celui d'une décision majoritaire parfaitement stupide — n'est guère plausible.

On pourrait à présent se demander dans quelle mesure tout cela est pertinent pour une défense épistémique de la démocratie. En quoi l'émergence d'une intelligence collective dans les contextes de résolution de problèmes et d'agrégation de jugementsimporte-t-elle? Ma thèse est que la résolution de problèmes décrit de manière adéquate la tâche qu'est censée accomplir la délibération, tandis que dans le même temps la prédiction collective décrit de manière adéquate la tâche quela règle de la majorité est le mieux à même d'effectuer. On pourrait sans doute affirmer que, dans la politique démocratique, la prise de décision collective consiste en une première délibération, au cours de laquelle on identifie les problèmes et propose des

11. Ces questions peuvent renvoyer à ce que Luppia (200I) appelle "les choix importants» (le type de questions d'orientation politique que l'on propose à l'occasion d'un référendum), mais également à des questions plus complexes et parfois même techniques, comme des questions économiques ou la réforme du système électoral, si du moins on a donné aux citoyens, avant le vote, du temps pour délibérer entre eux, car cela peut considérablement améliorer la qualité de leur opinion, en tout cas celle de son contenu informationnel (voir par exemple Fishkin, 2009). 
solutions. Suit alors une phase de vote, où l'on utilise la règle de la majorité pour déterminer laquelle des options proposées est la meilleure. Si tel est bien le cas, la combinaison des résultats que je viens de présenter permet de défendre un argument fort en faveur de l'intelligence démocratique, ou de ce que j'appelle "la raison démocratique».

Pour chaque procédure, que ce soit la délibération ou le vote, la diversité cognitive se révèle être d'une importance ou égale ou supérieure à l'aptitude individuelle moyenne. Dans le système cognitif « délibération + règle de la majorité ", la composante épistémique essentielle est celle de la diversité cognitive. L'aptitude individuelle moyenne a également son importance, mais seulement de manière secondaire. Quelle est à présent la manière la plus simple de maximiser la diversité cognitive au sein d'un groupe? Mon avis est que la manière la moins coûteuse et la plus simple d'assurer une grande diversité cognitive est d'inclure davantage de membres dans le groupe de ceux qui prennent les décisions. Cela demeure valide, ajouterais-je, même si inclure davantage de membres signifie en un sens faire baisser l'aptitude du membre moyen. En d'autres termes, s'il faut choisir entre accroître la participation à la prise de décision et réduire le réservoir des décideurs à une petite poignée de "meilleurs et plus brillants », le pari le moins risqué aussi contre-intuitif cela puisse-t-il paraître - consiste à miser sur le plus grand nombre.

Il en est ainsi parce que l'augmentation du nombre accroîtra naturellement la diversité cognitive. J'utilise le mot «naturellement» en me fondant sur l'hypothèse plausible (me semble-t-il) selon laquelle la diversité cognitive est quelque chose que l'on trouve dans n'importe quel groupe d'humains, puisque les gens viennent au monde pourvus de différentes boîtes à outils cognitives (voir les théories de l' "intelligence multiple », par exemple Gardner, I985; Sternberg, I985, Salovey and Meyer, I990). Il se peut que la distribution de ces types d'intelligences varie d'une société à l'autre, mais il paraît raisonnable de considérer qu'aucune société n'est constituée de manière monolithique d'individus pré-câblés percevant tous exactement le monde de la même manière. Quoi qu'il en soit, même des individus précâblés de manière similaire développeraient au fil du temps, sur la base de leurs propres expériences, des outils cognitifs, des cadres mentaux, des perspectives et des heuristiques uniques. En définitive, je pense qu'il est correct de considérer que, si elles ont la chance de développer librement des aptitudes innées distinctes, à long terme des personnes différentes finiront par voir le monde et approcher les problèmes de manière également très différente.

À l'opposé, essayer d'augmenter le «QI » du membre moyen du groupe de décideurs en sélectionnant un certain type de personnes aura probablement pour effet de réduire la diversité cognitive (car les personnes éduquées dans une même école et une même discipline ont tendance à penser de manière plus semblable). Plus important encore, même si rendre le processus 
de décision plus inclusif conduit à diminuer l'aptitude moyenne des membres du groupe, cette perte relative peut être plus que compensée par le gain de diversité cognitive. À l'inverse, le gain d'aptitude individuelle ne compensera pas nécessairement la perte de diversité cognitive. Cela s'explique une fois encore par le fait que, dans le cadre d'une prise de décision collective, la diversité cognitive a plus d'importance que l'aptitude individuelle pour l'émergence de l'intelligence du groupe. Autrement dit, il vaut souvent mieux sacrifier un certain taux d'aptitude moyenne en échange d'un montant équivalent de diversité cognitive, plutôt que de faire l'inverse.

On pourrait objecter que, même si elle est séduisante, l'idée d'inclure davantage de personnes se heurte à un problème de faisabilité au-delà d'un certain seuil. Bien qu'on puisse faire voter des millions d'individus, il est en effet impossible en pratique que tous les membres d'un groupe très large participent à la délibération. Au-delà d'un certain seuil numérique, la délibération se transforme en véritable chaos, auquel cas la supériorité épistémique semblerait aller par défaut à une délibération impliquant un plus petit nombre d'individus - et dans ce cas, on pourrait bien préférer s'en remettre aux plus intelligents ou aux plus cultivés. Cette objection est recevable, mais seulement dans une certaine mesure. Il y a sans doute un point de rupture au-delà duquel, quand davantage d'individus sont impliqués dans la délibération, on obtient des résultats de moindre qualité pour des raisons d'ordre purement pratique. Les sociétés ont heureusement trouvé depuis longtemps une solution à ce problème de seuil: la représentation. Ce qu'implique la théorie de l'intelligence collective, c'est que du point de vue épistémique le meilleur moyen de sélectionner des représentants n'est pas nécessairement d'avoir recours à des élections classiques, mais plutôt de procéder par tirage au sort. Si préserver la diversité cognitive compte effectivement davantage qu'élever l'aptitude individuelle moyenne des décideurs, alors procéder à une sélection par tirage au sort semblerait plus susceptible de conduire à des résultats intelligents qu'avoir recours à l'élection ${ }^{12}$.

Répétons-le: le point essentiel ici est que, si la diversité cognitive est l'élément clé de l'intelligence collective, alors, toutes choses étant égales par ailleurs, "plus» vaut épistémiquement mieux que «moins» ${ }^{13}$. Je propose ainsi de généraliser le «théorème de l'avantage de la diversité sur l'aptitude»

12. Il est bien entendu tout à fait possible, même si c'est peu vraisemblable, que la sélection par le moyen de l'élection préserve la diversité cognitive d'un groupe donné. Comme solution de rechange à un échantillon pris seulement au hasard, on peut proposer un échantillon également pris au hasard, mais stratifié: autrement dit, on fera une sélection au hasard parmi des groupes d'individus présentant un certain type de diversité. Cette dernière solution me laisse assez sceptique, partiellement en raison de la difficulté qu'il y a à définir les catégories sur la base desquelles l'échantillon devra être ainsi prélevé. Pour une exploration plus approfondie de ces questions, voir Landemore, 20I3.

13. Remarquons que, dans la mesure où (et si c'est bien le cas que) la diversité cognitive est corrélée à d'autres formes de diversité, comme le genre ou la diversité ethnique, l'argument 
en un "théorème de l'avantage du nombre sur l'aptitude", selon lequel ce qui importe le plus pour l'intelligence collective d'un groupe engagé dans une pratique délibérative, et de manière générale pour la démocratie, est le nombre de personnes que contient ce groupe. Cette idée que la diversité cognitive est corrélée au nombre ne sera pas toujours vérifiée, mais elle reste plus plausible que l'idée contraire, selon laquelle un groupe d'individus moins nombreux contiendrait davantage de diversité cognitive.

\section{Les implications de l'argument de l'intelligence collective}

Tournons-nous à présent vers les implications que l'on peut tirer de l'argument de l'intelligence collective. Il se peut que les plus nombreux soient plus intelligents que les moins nombreux, mais pourquoi doit-on en tenir compte? Et qui doit en tenir compte? Après tout, les démocrates sont déjà convaincus que la démocratie est une bonne chose. Dès lors, même si l'argument de l'intelligence collective peut les conforter dans leurs conclusions, il arrive néanmoins un peu après la bataille: les justifications fondées sur l'égalité, la justice, le consentement ou la liberté ont déjà fait tout le travail.

Sans doute l'argument de l'intelligence collective arrive-t-il trop tard pour défendre la cause de la démocratie. Bien des gens sont déjà morts au nom de l'égalité, du consentement, de la justice et de la liberté. Du point de vue historique, le recours à de telles valeurs s'est montré bien plus efficace, pour justifier le passage des systèmes monarchiques traditionnels à des formes de gouvernement démocratique, qu'un argument épistémique qui ne disposait, à cette époque, d'aucun fondement théorique, et encore moins empirique. Mais les sciences sociales sont aujourd'hui pourvues de moyens d'évaluer l'affirmation selon laquelle les démocraties sont des régimes relativement intelligents, par exemple en établissant une corrélation entre démocratie et paix (Weart, I998) ou entre démocratie et diminution des risques de famines (Sen, I999). Nous disposons également aujourd'hui de concepts (entre autres, celui d'intelligence collective) et de technologies (médias de masse, Internet, etc.) qui permettent de donner sens à l'idée que les plus nombreux sont plus intelligents que les moins nombreux. Autant du point de vue normatif que du point de vue du spécialiste des sciences sociales, il faut prendre au sérieux l'argument de l'intelligence collective, et ce pour un certain nombre de raisons.

Tout d'abord, l'argument de l'intelligence collective permet une explication fonctionnaliste de la légitimité de la démocratie, à la fois dans l'Antiquité et dans les deux cents dernières années. Une explication fonctionnaliste de ce genre consiste à montrer comment les effets épistémiques non intentionnels de la démocratie ont contribué, à long terme, à renforcer ce système sans que les acteurs impliqués n'en aient conscience, à titre de procédure privilégiée

laisse entendre que la discrimination positive est une bonne chose, pas seulement du point de vue de l'équité, mais aussi pour des raisons épistémiques. 
de prise de décision, et même si on l'avait d'abord mise en place pour des raisons non épistémiques (par exemple, pour des raisons d'équité) ${ }^{14}$. En d'autres termes, l'argument de l'intelligence collective complète les arguments intrinsèques fondés sur l'équité, la justice, le consentement ou d'autres valeurs démocratiques encore, et permet alors d'expliquer pourquoi, bien qu'elle ait d'abord été recherchée et instaurée au nom de ces valeurs, la démocratie a été maintenue comme un idéal viable. L'argument de l'intelligence collective pourrait même aujourd'hui expliquer pourquoi, en Occident du moins, il semble que nous fassions la transition vers des formes de gouvernance toujours plus démocratiques, avec ce que les critiques appellent de manière péjorative l'émergence de "démocraties du public» (Manin, 1995, Les principes du gouvernement démocratique), lesquelles, pourrait-on dire, constituent des formes de démocraties représentatives plus directes, plus inclusives, et peutêtre même, plus intelligentes. L'analyse fonctionnaliste rendue possible grâce à l'argument de l'intelligence collective n'est peut-être pas encore complète (il reste encore beaucoup de travail à accomplir pour comprendre le fonctionnement des «mécanismes» de la raison démocratique), mais c'est un début.

Deuxièmement, en tant qu'il relève d'une défense fonctionnaliste de la démocratie, l'argument de l'intelligence collective fournit un cadre théorique au travail des historiens et des spécialistes des sciences politiques quand ils s'efforcent d'établir des corrélations, sinon des liens de cause à effet, entre démocratie et développement (par exemple Przeworski, 2000), démocratie et paix (par exemple Weart, I998), démocratie et diminution du risque de famine (Sen, I999), démocratie et droits de l'homme ou baisse de la mortalité infantile, ou encore démocratie et capitalisation de la connaissance et de la production (Ober, 20I0). Développement, paix, faible mortalité infantile, connaissance, et de manière générale information exacte concernant le monde, tout cela constitue autant de critères indépendants des procédures, tels que les présuppose une approche épistémique de la démocratie. Si l'on pouvait démontrer empiriquement que les démocraties satisfont mieux à ces critères que les oligarchies ou les dictatures, on disposerait de justifications empiriques en faveur de l'argument de l'intelligence collective, lequel permettrait en retour d'unifier ces différentes entreprises empiriques ${ }^{15}$.

Enfin, sur le plan positif des sciences politiques, l'argument de l'intelligence collective nous invite à remettre en question la validité des conclusions

14. Pour une définition des analyses fonctionnalistes, voir Hardin, I980 et Elster, 2007.

15. À ce propos, l'argument de l'intelligence collective nous encourage également à faire le lien, non seulement entre différents types de littérature en théorie démocratique (en gros, la littérature normative et la littérature empirique), mais aussi entre les sciences politiques et d'autres disciplines encore. Les sciences politiques prennent généralement exemple sur l'économie, mais il y a tout un ensemble d'autres disciplines qui pourraient avoir un intérêt pour l'étude du comportement des votants ou celle des institutions démocratiques, parmi lesquelles les sciences cognitives et la théorie de l'information. 
auxquelles sont parvenus les chercheurs en matière d'opinion publique, quant à l'incompétence du public en général ${ }^{16}$. La recherche en matière d'opinion publique est peut-être qualifiée quand il s'agit de mesurer l'ignorance des individus (supposée équivalente à leur (in)compétence en matière politique), mais elle ne constitue pas un bon moyen de mesurer ce qui est en définitive un élément encore plus essentiel de l'intelligence collective, à savoir la diversité cognitive. On ne peut tirer de conclusions convaincantes à propos de l'incompétence démocratique à partir de mesures qui, au mieux, ne tiennent compte que d'un seul facteur dans l'équation de l'intelligence collective. Tant que la recherche en matière d'opinion publique ne sera pas capable de démontrer que le manque d'aptitude individuelle ne peut être compensé par la présence d'une diversité cognitive suffisante, la critique portée contre la prise de décision démocratique reposera sur des fondements insuffisants.

De tout cela, on peut tirer au moins trois implications pour la théorie normative de la démocratie. Premièrement, l'argument de l'intelligence collective nous invite à réévaluer la validité des arguments traditionnellement invoqués en faveur de la démocratie, à partir des idées de consentement individuel, de liberté, de justice ou d'égalité. S'il ne retire rien à la valeur de ces arguments en tant que tels, il permet néanmoins de fournir une analyse convaincante de ce qui fait la valeur de la démocratie à ceux qui ne croiraient pas déjà en la valeur du consentement, de la liberté et de l'égalité c'est-à-dire à quiconque ne partagerait pas déjà, à un degré ou un autre, la "foi en la démocratie» (Deneen, 2005). L'avantage de l'argument de l'intelligence collective est qu'il nous explique pourquoi il est instrumentalement bon d'inclure davantage de personnes dans le processus de décision, plutôt que d'exiger l'acceptation de ce principe d'inclusion en tant que tel, comme une question d'équité, de justice, ou de quelque autre valeur que ce soit. En ce sens, on peut dire que cet argument conforte les conclusions que l'on peut tirer des défenses purement intrinsèques de la démocratie, mais il leur donne un fondement plus utilitariste/instrumental/rationnel que ne pourrait le faire une simple croyance en la justice, l'égalité, le consentement ou la liberté. Une question demeure cependant sans réponse: celle de savoir si ces différents arguments opèrent en parallèle ou s'ils dépendent les uns des autres, et, dans ce dernier cas, s'ils se renforcent ou entrent en contradiction et se sapent les uns les autres. Plus haut, j'ai laissé entendre que l'argument de l'intelligence collective pouvait s'articuler aux arguments intrinsèques, au sein d'une analyse fonctionnaliste complète de la légitimité démocratique.

Une autre implication normative de l'argument de l'intelligence collective est qu'il remet en cause l'opposition traditionnelle que l'on fait entre les démocrates agrégatifs et les démocrates délibératifs, car la conception de la

16. Je renvoie ici à la littérature concernant les préférences éclairées, par exemple Althaus, 2003 et Caplan, 2007. 
démocratie sur lequel cet argument repose ne peut être correctement saisie par aucun de ces deux groupes. En effet, selon cet argument, la délibération n'est pas l'unique clé de voûte de la raison démocratique: il ne la considère que comme l'un des mécanismes d'un système démocratique qui est beaucoup plus large. L'argument de l'intelligence collective reconnaît en particulier la valeur et l'intérêt spécifique d'un mécanisme d'agrégation comme la règle de la majorité, qui permet de transformer une multiplicité de jugements individuels en prédictions collectives correctes, au lieu de ne la voir que comme un pis-aller par rapport à la délibération. De la même manière, l'argument de l'intelligence collective se distingue de la conception que l'on associe d'ordinaire à la démocratie agrégative, selon laquelle la politique ne porte que sur une agrégation d'intérêts et de préférences. L'argument de l'intelligence collective repose au contraire sur la conception des démocrates délibératifs selon laquelle la politique constitue aussi, si ce n'est avant tout, une recherche collective pour trouver des solutions répondant à des problèmes collectifs, et à la question de la justice. S’il doit y avoir agrégation, ce ne peut être qu'une agrégation de jugements et de prédictions, et non une agrégation d'intérêts.

Enfin, l'argument de l'intelligence collective permet de protéger les analyses de l'autorité politique qui font place à des considérations épistémiques contre ce que David Estlund appelle «le risque de l'épistocratie». D'après lui, en effet, "s'il existe un certain critère épistémique, alors il s'ensuit que certains savent mieux que d'autres, et que ce sont eux qui doivent gouverner, exactement comme dans le modèle aussi élégant que repoussant défendu par Platon dans La République» (Estlund, I997: I8). De même que permettre de discuter de la compétence des votants peut conduire à imposer des restrictions au droit de vote, partir du principe que l'autorité politique requiert un certain taux de succès épistémique pourrait nous encourager à déléguer les choix politiques à une caste de "savants» — qu'il s'agisse des rois-philosophes de Platon ou de tout autre type d'individus omniscients.

Si l'argument de l'intelligence collective est correct, nous ne courons cependant aucun risque de cette nature, puisqu'au regard de la plupart des questions politiques le groupe est vraisemblablement plus intelligent que n'importe quel sous-ensemble de ses membres défini a priori. En un mot: l'épistocratie n'est pas une option envisageable, non pas parce que nous ne pouvons jamais savoir qui sont les savants, ni parce qu'il n'y a aucun savant dont l'expertise ne puisse être remise en cause par les objections raisonnables des autres citoyens (ce qui constitue la réponse proposée par Estlund au défi de l'épistocratie), mais parce que le savant le plus fiable n'est autre en réalité que le groupe lui-même, par opposition à tout individu en particulier, ou tout groupe d'individus pris en son sein. Même s'il est probable que certains sous-ensembles d'experts s'y connaissent mieux sur certaines questions, toutes choses étant égales par ailleurs, le groupe en saura davantage 
sur l'ensemble des questions. Voilà pourquoi, abstraction faite de toutes les autres raisons que nous pouvons avoir de choisir la démocratie, la prise de décision démocratique constitue une stratégie plus sûre que toutes les versions envisageables de la règle du petit nombre.

\section{Conclusion}

Permettez-moi, en terminant, de dire un mot sur les implications politiques que l'argument de l'intelligence collective — s'il est correct — peut et devrait entraîner. J'ai mentionné précédemment que, si le but de la représentation est d'améliorer la qualité de la prise de décision collective sur le plan pratique, la sélection des représentants devrait se préoccuper davantage de préserver la diversité cognitive que d'élever le niveau de compétence individuelle des représentants - aussi contre-intuitif que cela puisse paraître. On pourrait objecter qu'il y a peut-être des manières plus intelligentes d'accroître la diversité cognitive que celle qui consiste à utiliser un échantillon choisi au hasard - par exemple en instaurant des quotas d'experts, sélectionnés dans différentes sous-catégories. Mais cela n'est vrai que si l'on part du principe que le type d'expertise dont on a besoin en politique peut être connu à l'avance. Sur ce point, je reprendrai à mon compte la réponse à la fois classique et convaincante que donnait le sophiste Protagoras au problème de la connaissance politique. Dans une discussion avec lui sur la raison d'être du principe de l'«iségorie» — le droit qu'ont tous les Athéniens de prendre la parole devant l'Assemblée - Socrate remarque que, s'il s'agit de construire un édifice public ou un vaisseau pour l'armée, les Athéniens non seulement laissent les charpentiers et les ingénieurs s'exprimer, mais font aussi taire les ignares. Cependant, lorsque la question concerne le bien public, c'est-à-dire les orientations politiques générales de la cité, tout le monde est invité à s'exprimer. Loin d'accepter la conclusion implicite de Socrate selon laquelle la politique n'est pas un objet d'enseignement ou de connaissance, le sophiste répond par un mythe qui suggère que chacun d'entre nous a en lui une partie de la capacité divine au jugement politique, et que nous sommes plus à même d'atteindre la vérité quand nous prenons les décisions en commun. Mon interprétation de la réponse du sophiste et de la pratique de l'iségorie est que, sur certaines questions, les Athéniens reconnaissent qu'il n'y a pas un seul expert que l'on puisse identifier a priori, à part le groupe lui-même.

Une autre conclusion évidente s'impose ici: chaque fois que c'est possible, il faudrait que la prise de décision collective soit plus inclusive et participative qu'elle ne l'est d'ordinaire. La question de la faisabilité constitue naturellement une contrainte majeure pour l'argument, en particulier dans les sociétés qui se caractérisent par la division massive qu'elles instaurent, au sein du travail cognitif, entre les politiciens professionnels et les citoyens ordinaires. La multiplication des assemblées locales (town-hall meetings) n'est peut-être plus nécessairement le meilleur moyen d'y parvenir, mais il existe de nouvelles formes de participation, comme celles qui ont été offertes 
récemment par l'initiative "Gouvernement ouvert ", et qui ouvrent de nouvelles perspectives d'expérimentation. Il nous reste encore à explorer la partie immergée de l'immense iceberg que constitue l'intelligence collective en politique.

$$
\text { * Traduit de l'anglais par Aude Bandini }
$$

\section{Bibliographie}

Althaus, Scott. Collective Preferences in Democratic Politics, Cambridge, Cambridge University Press, 2003.

Aristotle. Politics, traduit par C.D. Reeve, Indianapolis, Hackett Publishing, I998.

Caplan, Bryan. The Myth of the Rational Voter. Why Democracies Choose Bad Policies. Princeton, Princeton University Press, 2007.

Cohen, Joshua. "An Epistemic Conception of Democracy ", Ethics 97 (I), I986, 26-38.

Estlund, David. Democratic Authority: A Philosophical Framework, Princeton, Princeton University Press, 2008.

- "Beyond Fairness and Deliberation: The Epistemic Dimension of Democratic Authority» in J. Bohman and W. Rehg (eds), Deliberative Democracy. Essays on Reason and Politics, Cambridge MA, MIT Press, I997, I73-204.

Fishkin, James. When the People Speak: Deliberative Democracy and Public Consultation, Oxford, Oxford University Press, 2009.

Gardner, Howard. Frames of Mind: The Theory of Multiple Intelligences, New York, Basic, 1983 .

Goodin, Robert. Innovating Democracy: Democratic Theory and Practice After the Deliberative Turn, Oxford, Oxford University Press, 2008.

- Reflective Democracy, London, Oxford University Press, 2003.

Hardin, Russel. «Rationality, Irrationality and Functionalist Explanation», Social Science Information 19, I980, 755-772.

Hong, Lu and Scot Page. «Interpreted and Generated Signals ", Journal of Economic Theory I 44 (5), 2009, 2I 74-2I96. Ability Problem Solvers", Proceedings of the National Academy of Sciences, IOI (46), 2004, I6385-89.

—. "Problem Solving by Heterogeneous Agents", Journal of Economic Theory, 97 (I), 200I, I23-63.

Landemore, Hélène. Democratic Reason: Politics, Collective Intelligence, and the Rule of the Many, Princeton N. J., Princeton University Press, 20I3.

- «Democratic Reason: the Mechanisms of Collective Intelligence in Politics ", in H. Landemore and J. Elster, Collective Wisdom: Principles and Mechanisms. Cambridge, Cambridge University Press, 20I za.

- «Deliberation, Cognitive Diversity, and Democratic Inclusiveness: An Epistemic Argument for the Random Selection of Representatives ", Synthese DOI, 20I 2b, I0.I007/SII 229-0I 2-0062-6.

- Democratic Reason: Politics, Collective Intelligence, and the Rule of the Many (Ph.D. Dissertation, Harvard University), 2008.

Lupia, Arthur. "What We Should Know: Can Ordinary Citizens Make Extraordinary Choices? ", Unpublished Paper prepared for the WCFIA Conference on 
«Making Big Choices: Individual Opinion Formation and Societal Choice ", 2001 .

Mackay, Charles. Extraordinary Popular Delusions and the Madness of Crowds, I84I.

Manin, Bernard. The Principles of Representative Government, Cambridge, Cambridge University Press, I997.

Mill, John Stuart [I 86I]. Considerations on Representative Government, Cambridge, Cambridge University Press, 2010.

Ober, Josiah. Democracy and Knowledge: Innovation and Learning in Classical Athens. Princeton and Oxford, University of Princeton Press, 2010.

Page, Scott. The Difference. How the Power of Diversity Creates Better Groups, Firms, Schools, and Societies, Princeton, Princeton University Press, 2007.

Przeworski, Adam. Democracy and Development: Political Institutions and WellBeing in the World, Cambridge, NY, Cambridge University Press, 2000.

Rheingold, Howard. Smart Mobs, Cambridge, MA, Perseus Publishing, 2003. Traduction française: Foules intelligentes: la nouvelle révolution sociale, les éditions $\mathrm{M} 2 \mathrm{I}, 2005$.

Salovey, Peter and J.M. Meyer [1990]. «Emotional Intelligence» in Keith Oatley, Jennifer M. Jenkins, Nancy L. Stein, Human Emotions: A Reader, Oxford, Blackwell Publishers, I998, 313-320.

Sen, Amartya. Freedom as Development, Oxford, Oxford University Press, I999. Traduction française: Un nouveau modèle économique. Développement, justice, liberté, Paris, les éditions Odile Jacob, 2000.

Sternberg, Robert J. Beyond IQ: A Triarchic Theory of Human Intelligence, New York, Cambridge University Press, 1985.

Stich, Stephen. "Reflective Equilibrium, Analytic Epistemology and the Problem of Cognitive Diversity ", Synthese 74, I988, 39I-4I3.

Surowiecki, James. The Wisdom of Crowds. Why the Many are Smarter than the Few and How Collective Wisdom Shapes Business, Economies, Societies, and Nations. New York, London, Toronto, Sidney, Auckland, Doubleday, 2004. Traduction française: La sagesse des foules, les éditions Jean-Claude Lattès, Paris, 2008.

Sunstein, Cass. Infotopia: How Many Minds Produce Knowledge, Oxford, New York, Oxford University Press, 2006.

Waldron, Jeremy. "The Wisdom of the Multitude: Some Reflections on Book III Chapter II of the Politics ", Political Theory 23, I995, 563-584.

Weart, Spencer. Never At War: Why Democracies Will Not Fight One Another, New Haven, Yale University Press, I998. 